\title{
Class 1 heating cycles: A new class of thermodynamic cycles
}

\author{
Hong-Rui $\mathrm{Li}^{1,{ }^{*}}$ and Hua-Yu $\mathrm{Li}^{2,}{ }^{*}$ \\ ${ }^{1}$ Key Laboratory of Efficient Utilization of Low and Medium Grade Energy (Ministry \\ of Education), Tianjin University, Tianjin 300350, China. \\ ${ }^{2}$ Department of Energy and Power engineering, China University of Petroleum (East \\ China), Qingdao, Shandong 266580, China. \\ *Corresponding authors: 1hr@tju.edu.cn (H.-R.L.); lihy1963@upc.edu.cn (H.-Y.L.) \\ ORCID: 0000-0003-0249-7260 (H.-R.L.); 0000-0002-8229-019X (H.-Y.L.)
}

\begin{abstract}
Thermodynamic cycles are not only the core concepts of thermal science, but also key approaches to energy conversion and utilization. So far, power cycles and refrigeration cycles have been the only two general classes of thermodynamic cycles. While diverse types of systems have been developed to perform thermodynamic cycles, no new general classes of thermodynamic cycles have been proposed. Based on the basic principles of thermodynamics, here we propose and analyze a new general class of thermodynamic cycles named class 1 heating cycles (HC-1s). Two basic forms of $\mathrm{HC}-1 \mathrm{~s}$ are obtained by connecting six essential thermodynamic processes in the proper order and forming a thermodynamic cycle. $\mathrm{HC}-1 \mathrm{~s}$ present the simplest and most general approach to utilizing the temperature difference between a high-temperature heat source and a medium-temperature heat sink to achieve efficient medium-temperature heating and/or low-temperature cooling. HC-1s fill the gaps that have existed since the origin of thermal science, and they will play significant roles in
\end{abstract}


energy conservation and emission reduction.

\section{Keywords}

heating cycles; thermodynamic cycles; thermodynamics; temperature difference utilization; heating; cooling; cogeneration; thermal science

\section{Highlights}

- A new general class of thermodynamic cycles are constructed.

- Their nature, functions, characteristics and advantages are generally analyzed.

- A novel, efficient, coordinating, simple and general approach to heating and cooling.

- Only need six processes to achieve heat-driven heating and heat-driven cooling.

- Have advantages over current cogeneration, heat pump and absorption heat pump systems. 


\section{Main Text}

\section{Introduction}

\subsection{General classes of thermodynamic cycles}

Thermodynamic cycles are not only the core concepts of thermal science, but also essential to the global energy system. So far, power cycles and refrigeration cycles have been the only two general classes of thermodynamic cycles ${ }^{[1,2]}$. Power cycles (Figure 1a) utilize the temperature difference between a high-temperature heat source and a low-temperature heat sink to convert part of high-temperature heat into power; they are widely used in power plants, automobiles and aircraft. Refrigeration cycles (Figure 1b) utilize the grade difference between power and high-temperature heat to lift heat from a low temperature to a high temperature; they are widely used in refrigerators, air conditioners and heat pumps. These two general classes of thermodynamic cycles each present a simple and general approach to energy conversion and utilization.
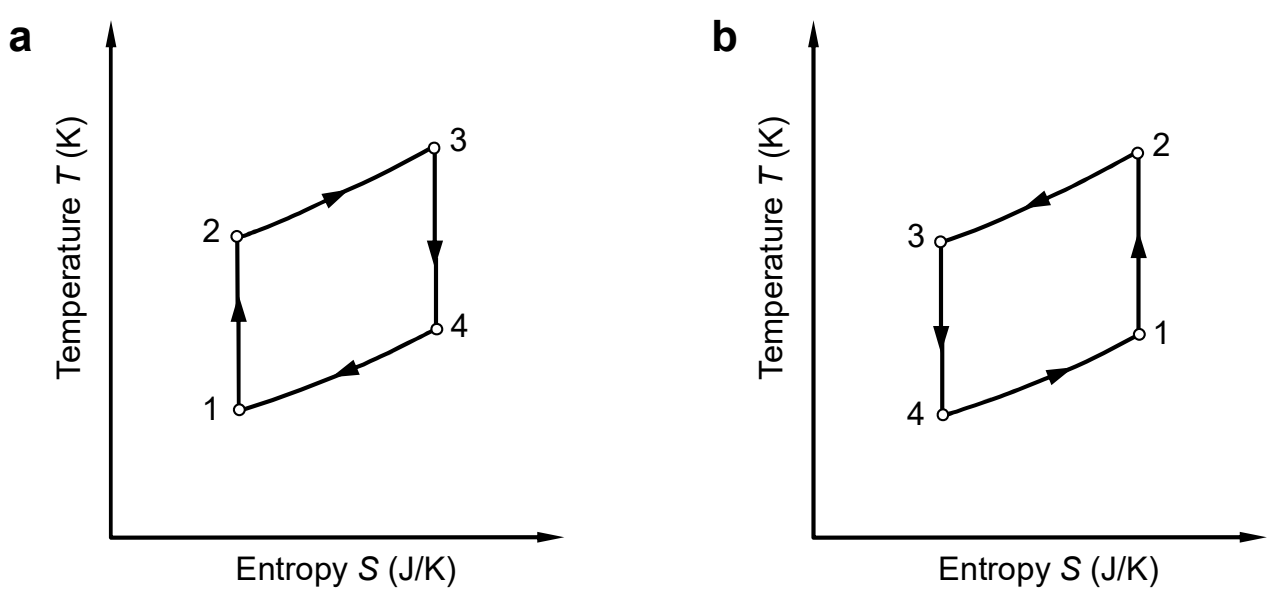

Figure 1. A basic power cycle and a basic refrigeration cycle. (a) A basic power cycle consists of four processes: pressurization 1-2, high-temperature heat 
absorption 2-3, depressurization 3-4, and low-temperature heat rejection 4-1. (b) A basic refrigeration cycle consists of four processes: pressurization 1-2, hightemperature heat rejection 2-3, depressurization 3-4, and low-temperature heat absorption 4-1.

The study of thermodynamic cycles has a long history. The early explorations of heat engines laid the technical foundations for studying thermodynamic cycles. In 1824, Sadi Carnot ${ }^{[3]}$ proposed the concept of a power cycle as a generalization of actual heat engines; he also reversed the power cycle to get a refrigeration cycle. Thereafter, scientists and engineers have been focusing on improving the performance of thermodynamic cycles, and exploring diverse means to perform thermodynamic cycles. Nowadays, the principles and methods of performance enhancement have been studied a lot; meanwhile, thermodynamic cycles can be performed by various types of systems, including the mechanical compression system ${ }^{[4,5]}$, electrochemical system ${ }^{[6]}$, thermoacoustic system ${ }^{[7]}$, magnetocaloric system ${ }^{[8]}$, electrocaloric system ${ }^{[9]}$, and twistocaloric system ${ }^{[10]}$. However, no new general classes of thermodynamic cycles have been proposed. Considering the importance of thermodynamic cycles in human life and production, two natural but rarely-raised questions arise: 1) Are there any new general classes of thermodynamic cycles? 2) If any, what are their functions, characteristics and advantages in energy conversion and utilization?

In this study, we propose a new general class of thermodynamic cycles named class 1 heating cycles (HC-1s). HC-1s present the simplest and most general approach to utilizing the temperature difference between a high-temperature heat source and a medium-temperature heat sink to achieve efficient medium-temperature heating and/or low-temperature cooling. HC-1s (more precisely, their basic forms) also 
provide the simplest operating procedure and equipment structure for heat-driven heating and heat-driven cooling. Without loss of generality (and as most thermodynamics textbooks do), we mainly introduce and discuss HC-1s on the basis of the mechanical compression system, which is the most common and intensivelystudied means to perform thermodynamic cycles.

\subsection{The sustainable utilization and production of thermal energy}

Considering that approximately $90 \%$ of the global energy consumption involves the generation or manipulation of heat, achieving the sustainable utilization and production of heat is vital for solving global energy and environmental problems ${ }^{[11]}$. It is worth noting that there is often a huge temperature difference between heat supply and heat demand. The most typical case in which such a temperature difference exists is burning fossil fuels for heating, which is the main method for humans to obtain heat. The temperature of the heat released from burning fossil fuels can exceed $1,400^{\circ} \mathrm{C}$; in contrast, the temperature of the heat required for residential heating is lower than $100^{\circ} \mathrm{C}$, and it is estimated that $52.6 \%$ of European industrial heat demand is below $400^{\circ} \mathrm{C}^{[12]}$. This temperature difference may also exist when people use nuclear energy, biomass energy, solar energy or hydrogen energy for heating. However, people have long been accustomed to simply and directly transferring hightemperature heat to medium-temperature heat consumers without utilizing the temperature difference between the two; this conventional heating method irreversibly reduces the energy quality by a large margin and has contributed to a series of serious problems, including high energy costs, climate change ${ }^{[13]}$, environmental pollution and health crisis ${ }^{[14]}$. Researchers have proposed several advanced heating technologies, including cogeneration (also called combined heat and power, CHP) ${ }^{[15-}$ 
${ }^{17]}$, heat pumps ${ }^{[5]}$ and adsorption heat pumps ${ }^{[18]}$, to replace the conventional heating method; however, the former two are confined to the improvement or combination of power cycles and refrigeration cycles, and the latter rely on the special characteristics of solutions and thus lack generality. In contrast, we aim at achieving efficient heating from the higher perspective of constructing a new general class of thermodynamic cycles.

\section{Construction}

We construct HC-1s on the account of the following logic. Consider the simplest case: high-temperature heat is the only energy supply, and mediumtemperature heat is the only energy demand - thermodynamically, they correspond to a high-temperature heat source and a medium-temperature heat sink respectively. First, we can easily find a low-temperature heat source-usually the environment or a low-temperature heat resource can play this role. We intend to utilize the temperature difference between the high-temperature heat source and the medium-temperature heat sink, to overcome the temperature difference between the low-temperature heat source and the medium-temperature heat sink. Simply speaking, the core task of HC1s is to move high-temperature heat and low-temperature heat together to the medium temperature. As a result, the medium-temperature heat sink obtains more heat than that provided by the high-temperature heat source, so efficient heating is achieved.

Then, we explore the minimum number of steps required to achieve this task. Since an HC-1 operates among a high-temperature heat source, a medium-temperature heat sink and a low-temperature heat source, it contains at least three heat transfer processes. Besides, in order to switch circularly among three temperature levels, the 
cycle requires at least three transition processes (in the mechanical compression system, transition processes are often pressurization and depressurization processes), so the cycle contains a total of at least six thermodynamic processes.

Finally, the basic forms of HC-1s can be obtained by connecting these six essential processes in the proper order and forming a thermodynamic cycle. We notice that the cycle's three transition processes can be carried out in two different relative orders - the cycle's working medium can either first transition from the low temperature to the high temperature, then to the medium temperature, and finally to the low temperature; or it can first transition from the low temperature to the medium temperature, then to the high temperature, and finally to the low temperature. Correspondingly, HC-1s have two basic forms:

The basic form A of HC-1s (HC-1A, Figure 2a) consists of the following six processes: pressurization 1-2, high-temperature heat absorption 2-3, depressurization 3-4, medium-temperature heat rejection 4-5, depressurization 5-6, and lowtemperature heat absorption 6-1.

The basic form B of HC-1s (HC-1B, Figure 2b) consists of the following six processes: pressurization 1-2, medium-temperature heat rejection 2-3, pressurization 3-4, high-temperature heat absorption 4-5, depressurization 5-6, and low-temperature heat absorption 6-1. 
a

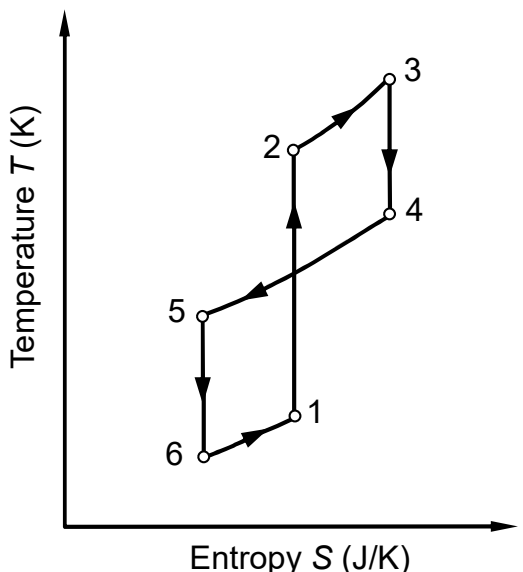

b

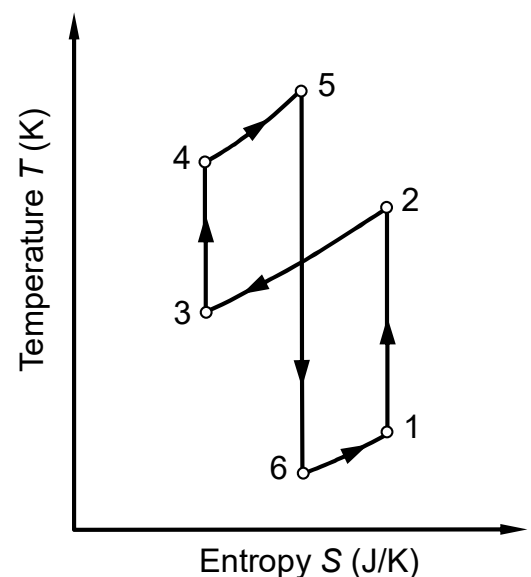

Figure 2. The basic forms of class 1 heating cycles (HC-1s). (a) An HC-1A consists of six processes: pressurization 1-2, high-temperature heat absorption 23, depressurization 3-4, medium-temperature heat rejection 4-5, depressurization 5-6, and low-temperature heat absorption 6-1. Although process 1-2 and process 4-5 seem to intersect in the temperature-entropy $(T-S)$ diagram, they are independent of each other. (b) An HC-1B consists of six processes: pressurization 1-2, medium-temperature heat rejection 2-3, pressurization 3-4, hightemperature heat absorption 4-5, depressurization 5-6, and low-temperature heat absorption 6-1. Process 2-3 and process 5-6 are also independent of each other.

If needed, adjustments or improvements can be applied to these basic forms to obtain diverse cycle structures, and thus better performance can be achieved without losing simplicity. Therefore, although we only show their two basic forms, HC-1s are actually a huge family of thermodynamic cycles, just like power cycles and refrigeration cycles. 


\section{Analysis}

Next, we analyze the functions, characteristics and advantages of $\mathrm{HC}-1 \mathrm{~s}$ based on their basic forms.

\subsection{The basic functions of $\mathrm{HC}-1 \mathrm{~s}$}

It is worth noting that the logic in Section 2 can also be transferred to the case of utilizing heat for cooling; in this case, a cold consumer serves as the lowtemperature heat source, usually the environment serves as the medium-temperature heat sink, and an object hotter than the environment serves as the high-temperature heat source. In the best case, a heat consumer serves as the medium-temperature heat sink while a cold consumer serves as the low-temperature heat source, then an HC-1 can satisfy both by utilizing high-temperature heat. In addition, if there exists a power demand or a power supply (although it is not considered in the above analysis), an HC-1 can also produce or utilize power efficiently.

According to the law of conservation of energy, we have

$$
W_{\text {net }}=Q_{\text {in, } \mathrm{H}}+Q_{\mathrm{in,L}}-Q_{\text {out }}
$$

where $W_{\text {net }}$ is the net work output of an $\mathrm{HC}-1, Q_{\mathrm{in}, \mathrm{H}}$ is the amount of heat absorbed from the high-temperature heat source by the cycle, $Q_{\mathrm{in}, \mathrm{L}}$ is the amount of heat absorbed from the low-temperature heat source by the cycle, and $Q_{\text {out }}$ is the amount of heat rejected to the medium-temperature heat sink by the cycle.

For an HC-1 with $W_{\text {net }}=0$, Eq. (1) can be written as $Q_{\text {out }}=Q_{\mathrm{in}, \mathrm{H}}+Q_{\mathrm{in}, \mathrm{L}}$. The cycle utilizes the temperature difference between the high-temperature heat source and the medium-temperature heat sink, to efficiently supply medium-temperature heat $Q_{\text {out, }}$ or low-temperature cold energy (by absorbing $Q_{\text {in, }}$ ), or both. 
For an HC-1 with $W_{\text {net }}>0$, Eq. (1) can be written as $Q_{\text {out }}+W_{\text {net }}=Q_{\text {in, } \mathrm{H}}+Q_{\text {in, } \mathrm{L}}$. The cycle utilizes the above-mentioned temperature difference to efficiently supply medium-temperature heat $Q_{\text {out }}$ (or supply low-temperature cold energy by absorbing

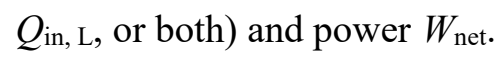

For an HC-1 with $W_{\text {net }}<0$, Eq. (1) can be written as $Q_{\text {out }}=Q_{\mathrm{in}, \mathrm{H}}+Q_{\mathrm{in}, \mathrm{L}}+\left|W_{\text {net }}\right|$. The cycle utilizes the above-mentioned temperature difference, as well as the grade difference between power and medium-temperature heat, to efficiently supply medium-temperature heat $Q_{\text {out }}$, or low-temperature cold energy (by absorbing $Q_{\text {in, L }}$ ), or both.

For an internally reversible $\mathrm{HC}-1, W_{\text {net }}$ can be expressed as the difference between the areas enclosed by the cycle's clockwise part and the anticlockwise part in the $T-S$ diagram. Here we take an HC-1A as an example to prove this statement schematically (Figure 3).

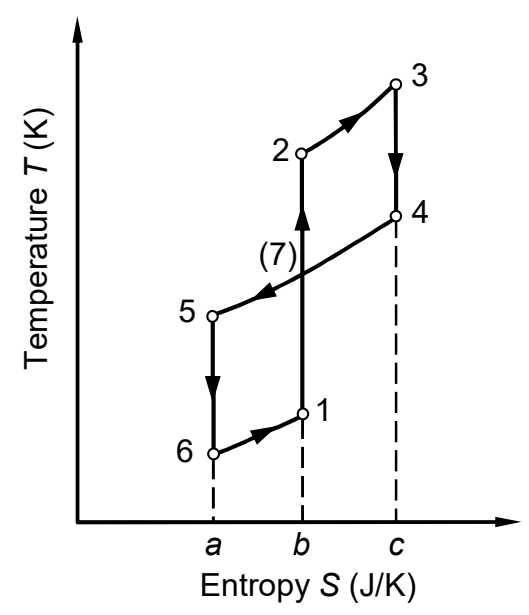

Figure 3. The auxiliary figure for proving the statement that the net work output of an internally reversible $\mathrm{HC}-1$ can be expressed as the difference between the areas enclosed by the cycle's clockwise part and the anticlockwise part in the T-S 
diagram. Both process 1-2 and process 4-5 pass through state point 7, which means this state exists in both processes but the two processes are independent of each other. The area $(A)$ under the curve of each internally reversible heat transfer process represents the amount of heat transfer for that process ${ }^{[1]}$.

The amounts of the cycle's heat transfer can be expressed as

$$
\begin{aligned}
Q_{\text {in, } \mathrm{H}} & =A_{b-2-3-c-b} \\
Q_{\text {in, } \mathrm{L}} & =A_{a-6-1-b-a} \\
Q_{\text {out }} & =A_{a-5-4-c-a}
\end{aligned}
$$

Thus,

$$
\begin{aligned}
W_{\text {net }} & =Q_{\mathrm{in}, \mathrm{H}}+Q_{\mathrm{in}, \mathrm{L}}-Q_{\mathrm{out}} \\
& =A_{b-2-3-c-b}+A_{a-6-1-b-a}-A_{a-5-4-c-a} \\
& =A_{b-2-3-c-b}+A_{a-6-1-b-a}-\left(A_{a-5-7-b-a}+A_{b-7-4-c-b}\right) \\
& =\left(A_{b-2-3-c-b}-A_{b-7-4-c-b}\right)-\left(A_{a-5-7-b-a}-A_{a-6-1-b-a}\right) \\
& =A_{7-2-3-4-7}-A_{6-5-7-1-6} \\
& =A_{\text {clockwise }}-A_{\text {anticlockwise }}
\end{aligned}
$$

The proof is complete. Therefore, for an internally reversible $\mathrm{HC}-1$ with $W_{\text {net }}=0$, $W_{\text {net }}>0$, or $W_{\text {net }}<0$, the area enclosed by the cycle's clockwise part is equal to, larger than, or smaller than that enclosed by the anticlockwise part in the $T-S$ diagram, respectively. This conclusion provides a visual guidance for designing the shapes and parameters of $\mathrm{HC}-1 \mathrm{~s}$ to meet diverse energy supplies and demands.

\subsection{Coordinating: HC-1s vs power cycles}

We can obtain the advantages of $\mathrm{HC}-1 \mathrm{~s}$ by comparing them with the former two general classes of thermodynamic cycles - power cycles and refrigeration cycles (and their corresponding heating methods). Both $\mathrm{HC}-1 \mathrm{~s}$ and power cycles can utilize 
high-temperature heat, but the two differ a lot in energy products. The core function of $\mathrm{HC}-1 \mathrm{~s}$ is to utilize high-temperature heat for heating and/or cooling; besides, $\mathrm{HC}-1 \mathrm{~s}$ can also produce or utilize power according to the local demand and supply of energy. In contrast, the core function of power cycles is to utilize high-temperature heat for power generation; heating can only be the by-product of power cycles, and cooling even cannot be produced by power cycles. Therefore, HC-1s show significant coordinating ability in the situations where heating or cooling is the foremost or even sole energy demand, such as oil processing, food processing and residential heating, because their products satisfy local demands well. In contrast, most current cogeneration systems are based on power cycles, and they have to use hightemperature heat to firstly generate power then supply heat ${ }^{[15]}$; therefore, the heat and power produced by these systems have to meet certain proportions, otherwise these systems will be inefficient (the similar problem also occurs in non-cyclic cogeneration systems, e.g., fuel-cell-based systems and solar photovoltaic/thermal systems). The ratio of the heat to the power produced varies from 1.4 to 2.6 for different types of current cogeneration systems ${ }^{[16]}$. If too much power is produced to be consumed locally, the user of a current cogeneration system has to export the redundant power to the grid ${ }^{[17]}$; in this case, the system becomes complex and the initial investment is compelled to rise. Moreover, even if the user only needs heating, the cogeneration system still has to produce power and thus will require more fuel than the conventional method of direct heating; in contrast, an $\mathrm{HC}-1$ in such a situation will consume less fuel than direct heating, because when $W_{\text {net }}=0$ we have $Q_{\text {out }}>Q_{\text {in, H }}$. Less fuel means lower local emission and lower transportation costs.

\subsection{Simplicity: HC-1s vs refrigeration cycles}


Both HC-1s and refrigeration cycles can achieve efficient heating and cooling, but the two differ a lot in energy inputs. The working principle of refrigeration cycles is utilizing power to lift heat, so they can only be used in the situations where power supply exists. However, power is usually a secondary energy source and mostly generated by power cycles. Therefore, although a heat pump ${ }^{[5]}$ or a refrigerator itself operates on a refrigeration cycle, people actually need a combination of a power cycle and a refrigeration cycle to achieve efficient heating or cooling - the power cycle coverts part of heat into power, then the power is transmitted to the refrigeration cycle, and finally the refrigeration cycle utilize the power to lift heat. The power cycle either operates between the above-mentioned high-temperature heat source and medium-temperature heat sink (Figure 4b, cycle 1-2-3-4-1), or is located in a power plant elsewhere; the refrigeration cycle operates between the above-mentioned medium-temperature heat sink and low-temperature heat source (Figure 4b, cycle 5-67-8-5). Since a basic power cycle and a basic refrigeration cycle each contain four thermodynamic processes, the combination of both requires eight thermodynamic processes and a power transmission process between two cycles. In contrast, a basic HC-1 only needs six thermodynamic processes to achieve the same goal, and it also avoids the transmission loss between two cycles. Moreover, HC-1s can be further simplified in special cases. For example, when heating up a gas which is suitable to be the cycle's working medium, we can employ it to directly conduct an open cycle, i.e., 5-6-1-2-3-4 in Figure 2a or 3-4-5-6-1-2 in Figure 2b. We can get the heated gas at the exit of the open cycle with no need for the process of medium-temperature heat rejection and the corresponding heat exchanger. 

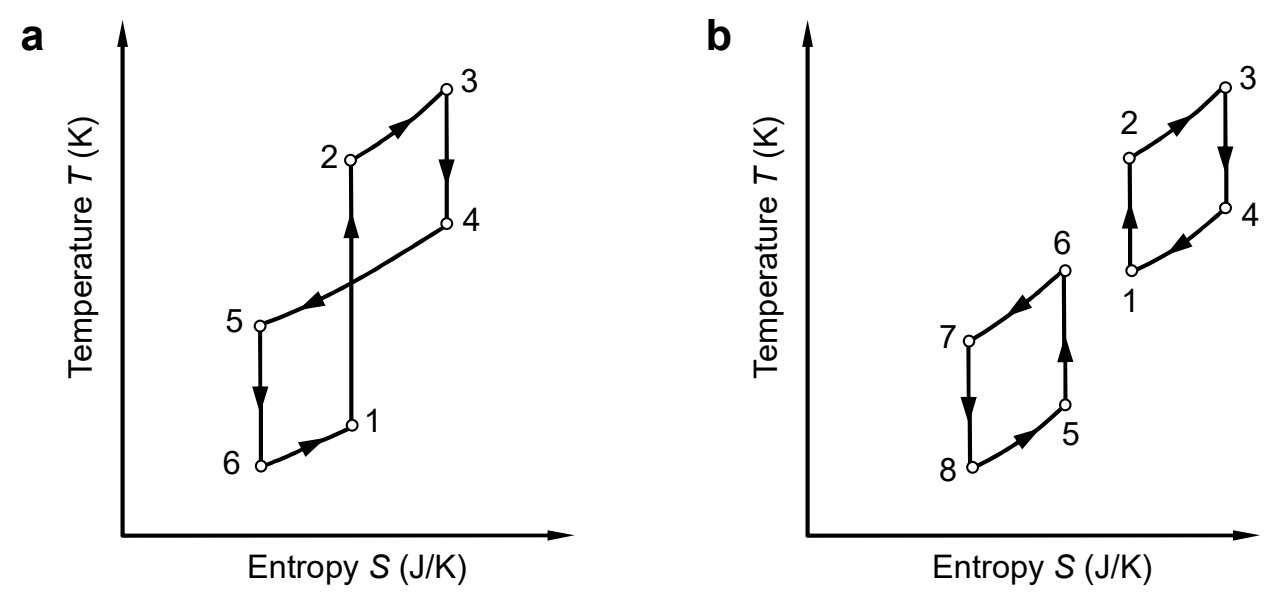

Figure 4. The comparison between an HC-1 and the combination of a power cycle and a refrigeration cycle. (a) A basic $\mathrm{HC}-1$ only requires one cycle with six thermodynamic processes. (b) The combination of a basic power cycle and a basic refrigeration cycle requires two cycles with eight thermodynamic processes (and a power transmission process between two cycles) in total. The power cycle 1-2-3-4-1 operates between the high-temperature heat source and the mediumtemperature heat sink; the refrigeration cycle 5-6-7-8-5 operates between the medium-temperature heat sink and the low-temperature heat source; the power cycle drives the refrigeration cycle.

\subsection{Generality: HC-1s vs absorption heat pumps}

We only use the fundamentals of thermodynamics in the construction of $\mathrm{HC}$ 1s without involving characteristics of any specific working medium. Therefore, HC1s can be performed by various working media, and the range of their achievable operating temperatures is as wide as that of current thermodynamic cycles.

Furthermore, although we mainly describe HC-1s on the basis of the mechanical compression system, we infer that they can also be performed by other types of 
systems with their functions and advantages remained; to this end, the processes of pressurization and depressurization mentioned above should be replaced with the corresponding transition processes in each system. In contrast, the common heatdriven heating technology and heat-driven cooling technology, i.e., absorption heat pumps and absorption chillers ${ }^{[18]}$, have to operate on the concentration change of a solution. Thus, they face restrictions in many aspects, including the choice of their working media and materials, as well as the range of their operating temperatures and sizes; besides, their working mechanism lacks generality and thus is hard be transferred to other types of systems.

\section{Examples}

Here we show three typical examples of HC-1s (without loss of generality, they are all in basic form A); they each possess clear theoretical significance or practical value. We also present their coefficients of performance (COPs, the ratio of the cycle's output to input) for medium-temperature heating or low-temperature cooling when $W_{\text {net }}=0$; the derivation is included in the Supplementary Information. 
a

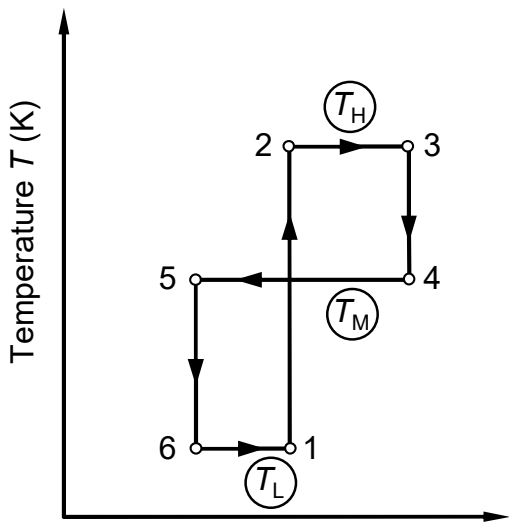

Entropy $S(\mathrm{~J} / \mathrm{K})$

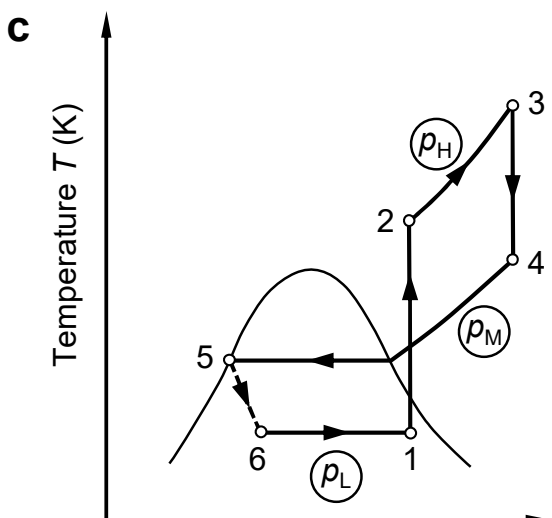

Entropy $S(\mathrm{~J} / \mathrm{K})$ b

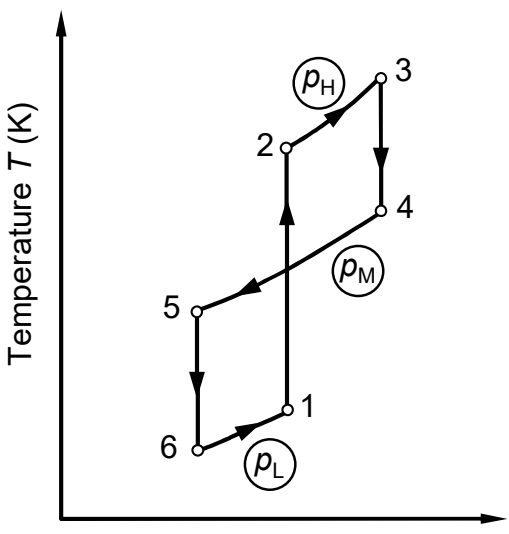

Entropy $S(\mathrm{~J} / \mathrm{K})$

Figure 5. Three typical examples of HC-1s. (a) An HC-1A with isothermal heat transfer processes. $T_{H}, T_{M}$ and $T_{L}$ are the working medium's thermodynamic temperatures during high-temperature heat absorption, medium-temperature heat rejection, and low-temperature heat absorption, respectively. (b) An HC-1A with isobaric heat transfer processes, employing an ideal gas as its working medium. (c) An HC-1A with isobaric heat transfer processes, employing a phasechange working medium. The depressurization (throttling) process 5-6 is internally irreversible and thus expressed as a dotted line. In (b) and (c), $p_{\mathrm{H},} p_{\mathrm{M}}$ and $p_{L}$ are the working medium's pressures during high-temperature heat absorption, medium-temperature heat rejection, and low-temperature heat 
absorption, respectively.

\subsection{The HC-1A with isothermal heat transfer processes}

The first example (Figure 5a) has isothermal heat transfer processes so it can clearly reveal the proportional relationships during moving heat among three different temperatures. Regarding the cycle as internally reversible ${ }^{[1]}$, we can express its COPs as

$$
\begin{aligned}
& \operatorname{COP}_{\mathrm{H}}=\frac{Q_{\text {out }}}{Q_{\mathrm{in}, \mathrm{H}}}=\frac{T_{\mathrm{M}}\left(T_{\mathrm{H}}-T_{\mathrm{L}}\right)}{T_{\mathrm{H}}\left(T_{\mathrm{M}}-T_{\mathrm{L}}\right)} \\
& \mathrm{COP}_{\mathrm{C}}=\frac{Q_{\mathrm{in}, \mathrm{L}}}{Q_{\mathrm{in}, \mathrm{H}}}=\frac{T_{\mathrm{L}}\left(T_{\mathrm{H}}-T_{\mathrm{M}}\right)}{T_{\mathrm{H}}\left(T_{\mathrm{M}}-T_{\mathrm{L}}\right)}
\end{aligned}
$$

where $\mathrm{COP}_{\mathrm{H}}$ and $\mathrm{COP}_{\mathrm{C}}$ are the cycle's COPs for medium-temperature heating and low-temperature cooling respectively.

\subsection{The HC-1A with isobaric heat transfer processes, employing an ideal gas as its working medium}

The other two are not hard to be realized on the basis of current technologies. The second example (Figure 5b) has isobaric heat transfer processes and employs an ideal gas as its working medium. Regarding the cycle as internally reversible, and the ideal gas's isobaric specific heat as constant ${ }^{[1]}$, we can express the cycle's COPs as

$$
\operatorname{COP}_{\mathrm{H}}=\frac{Q_{\text {out }}}{Q_{\mathrm{in}, \mathrm{H}}}=\frac{p_{\mathrm{M}}^{\frac{k-1}{k}}\left(p_{\mathrm{H}}^{\frac{k-1}{k}}-p_{\mathrm{L}}^{\frac{k-1}{k}}\right)}{p_{\mathrm{H}}^{\frac{k-1}{k}}\left(p_{\mathrm{M}}^{\frac{k-1}{k}}-p_{\mathrm{L}}^{\frac{k-1}{k}}\right)}
$$




$$
\mathrm{COP}_{\mathrm{C}}=\frac{Q_{\mathrm{in}, \mathrm{L}}}{Q_{\mathrm{in}, \mathrm{H}}}=\frac{p_{\mathrm{L}}^{\frac{k-1}{k}}\left(p_{\mathrm{H}}^{\frac{k-1}{k}}-p_{\mathrm{M}}^{\frac{k-1}{k}}\right)}{p_{\mathrm{H}}^{\frac{k-1}{k}}\left(p_{\mathrm{M}}^{\frac{k-1}{k}}-p_{\mathrm{L}}^{\frac{k-1}{k}}\right)}
$$

where $k$ is the ideal gas's specific heat ratio.

\subsection{The HC-1A with isobaric heat transfer processes, employing a phase-change working medium}

The third example (Figure 5c) has isobaric heat transfer processes and employs a phase-change working medium. The cycle's COPs can be expressed as

$$
\begin{aligned}
& \mathrm{COP}_{\mathrm{H}}=\frac{Q_{\text {out }}}{Q_{\text {in, } \mathrm{H}}}=\frac{h_{4}-h_{5}}{h_{3}-h_{2}} \\
& \mathrm{COP}_{\mathrm{C}}=\frac{Q_{\mathrm{in}, \mathrm{L}}}{Q_{\mathrm{in}, \mathrm{H}}}=\frac{h_{1}-h_{6}}{h_{3}-h_{2}}
\end{aligned}
$$

where $h$ is the working medium's enthalpy per unit of mass at each state point.

\section{Conclusions}

Each thermodynamic process of $\mathrm{HC}-1$ s has been widely used in practice and exists in every current power cycle and refrigeration cycle; besides, all the functions, characteristics and advantages of HC-1s are demonstrated by analysis on the basis of the basic principles of thermodynamics. Therefore, neither numerical nor experimental case studies are required to reach the conclusions of this article (but they can be future research contents). Theoretically, HC-1s are in equal status with power cycles and refrigeration cycles, and they fill the gaps that have existed since the origin of thermal science. Practically, HC-1s provide a novel, efficient, coordinating, simple 
and general approach to heating and cooling, and they will play significant roles in energy conservation and emission reduction. Furthermore, considering that the concept of thermodynamic cycles has been transferred and extended to other fields (e.g., chemistry ${ }^{[19,20]}$ and meteorology ${ }^{[21,22]}$ ), HC-1s are expected to offer profound inspirations to these fields. 


\section{Acknowledgements}

We thank Prof. Li Zhao for discussions of the manuscript.

\section{Competing interests}

H.-Y.L. is the inventor and applicant of the following patents on HC-1s: CN ZL201610244166.7; CN ZL201610244136.6; CN ZL201610240686.0; CN

ZL201610240615.0; all the patents have been authorized. H.-Y.L. is the main founder of two energy technology companies.

\section{Author contributions}

H.-Y.L. proposed the initial concept. H.-R.L. and H.-Y.L. performed the analysis. H.R.L. and H.-Y.L. wrote the manuscript. H.-R.L. drew the figures and derived the formulae.

\section{Supplementary Information}

Supplementary Information is available for this paper. 


\section{References}

[1] Çengel YA, Boles MA, Kanoğlu M. Thermodynamics: An engineering approach. 9 ed. New York: McGraw-Hill Education, 2019.

[2] Moran MJ, Shapiro HN, Boettner DD, et al. Fundamentals of engineering thermodynamics. 8 ed. Wiley, 2014.

[3] Carnot S. Reflections on the motive power of fire, and on machines fitted to develop that power. Paris: Bachelier, 1824.

[4] Chen H, Goswami DY, Stefanakos EK. A review of thermodynamic cycles and working fluids for the conversion of low-grade heat. Renewable Sustainable Energy Rev, 2010, 14: 3059-3067.

[5] Chua KJ, Chou SK, Yang WM. Advances in heat pump systems: A review. Appl Energy, 2010, 87: 3611-3624.

[6] Poletayev AD, McKay IS, Chueh WC, et al. Continuous electrochemical heat engines. Energy Environ Sci, 2018, 11: 2964-2971.

[7] Backhaus S, Swift GW. A thermoacoustic stirling heat engine. Nature, 1999, 399: $335-338$

[8] Romero Gómez J, Ferreiro Garcia R, De Miguel Catoira A, et al. Magnetocaloric effect: A review of the thermodynamic cycles in magnetic refrigeration. Renewable Sustainable Energy Rev, 2013, 17: 74-82.

[9] Ma R, Zhang Z, Tong K, et al. Highly efficient electrocaloric cooling with electrostatic actuation. Science, 2017, 357: 1130-1134.

[10]Wang R, Fang S, Xiao Y, et al. Torsional refrigeration by twisted, coiled, and supercoiled fibers. Science, 2019, 366: 216-221. 
[11]Henry A, Prasher R, Majumdar A. Five thermal energy grand challenges for decarbonization. Nat Energy, 2020, 5: 635-637.

[12]Naegler T, Simon S, Klein M, et al. Quantification of the european industrial heat demand by branch and temperature level. Int J Energy Res, 2015, 39: 2019-2030.

[13]IPCC. Climate change 2014: Mitigation of climate change. Contribution of working group iii to the fifth assessment report of the intergovernmental panel on climate change. Cambridge University Press, 2014.

[14]Lelieveld J, Evans JS, Fnais M, et al. The contribution of outdoor air pollution sources to premature mortality on a global scale. Nature, 2015, 525: 367-371.

[15] Onovwiona HI, Ugursal VI. Residential cogeneration systems: Review of the current technology. Renewable Sustainable Energy Rev, 2006, 10: 389-431.

[16] Strachan N, Farrell A. Emissions from distributed vs. Centralized generation: The importance of system performance. Energy Policy, 2006, 34: 2677-2689.

[17]Pilavachi PA. Mini- and micro-gas turbines for combined heat and power. Appl Therm Eng, 2002, 22: 2003-2014.

[18]Herold KE, Radermacher R, Klein SA. Absorption chillers and heat pumps. 2 ed. CRC Press, 2016.

[19]Gordon JM, Orlov VN. Performance characteristics of endoreversible chemical engines. J Appl Phys, 1993, 74: 5303-5309.

[20]Brogioli D. Extracting renewable energy from a salinity difference using a capacitor. Phys Rev Lett, 2009, 103: 058501.

[21]Emanuel KA. An air-sea interaction theory for tropical cyclones. Part i: Steadystate maintenance. J Atmos Sci, 1986, 43: 585-605. 
[22]Laliberté F, Zika J, Mudryk L, et al. Constrained work output of the moist atmospheric heat engine in a warming climate. Science, 2015, 347: 540-543. 